\title{
CONE BEAM COMPUTED TOMOGRAPHY THREE DIMENSIONAL RECONSTRUCTED IMAGES VERSUS SYNTHESIZED TWO DIMENSIONAL LATERAL IMAGES FOR THE EVALUATION OF THE NASOMAXILLARY COMPLEX IN PATIENTS WITH CLEFT LIP AND PALATE
}

\author{
Dina Hussein El-Ghoul*
}

\begin{abstract}
Introduction: The aim of this study was to compare the angular and linear measurements of the nasomaxillary complex for unilateral cleft lip and palate patients obtained from CBCT synthesized two dimensional lateral cephalograms to those from CBCT three dimensional reconstructed images.

Methods: This study comprised 10 children (6 boys and 4 girls) with surgically repaired complete unilateral cleft lip and palate (UCLP). Seven points were created and identified on the three dimensional volumetric model. The identified landmarks were then refined in the coronal, sagittal and axial tomographic slices to insure the right position of the points. Three angular and three linear measurements were determined and calculated by the software.
\end{abstract}

Results: The results of this study shows a close agreement between all the two dimensional and three dimensional measurements with lesser agreement regarding the Max-Height.

Conclusions: By constructing a perspective lateral image from CBCT scan, one can replicate the inherent magnification of a conventional two dimensional lateral cephalogram with high accuracy. This image can be used in place of a two dimensional lateral cephalogram for comparison with either normative data or serial records.

\section{INTRODUCTION}

Clefts of the lip and / or palate are one of the most common congenital craniofacial deformities. These anomalies have multifactorial inheritance, with both genetics and environment making varying contributions, and they can occur as a feature of various genetically determined syndromes. ${ }^{(1)}$

Craniofacial morphology of children with clefts

\footnotetext{
* Lecturer, Orthodontic Department. Faculty of Oral and Dental Medicine, Ain Shams University, Cairo, Egypt.
} 
has been reported to differ from that of normal children. The changes are due to mechanical presence of the cleft and lack of continuity of the tissues, lip and / or palate, or a combination of these factors. ${ }^{(2)}$ Treating these malformations requires a number of early surgical procedures that influence future maxillary growth ${ }^{(3)}$

Following lip and palate surgery during childhood, the resulting muscular forces and fibrosis alter future growth of the jaw, causing constriction of the maxillary dental arch. Theses post- operative morphological alterations result in a medial displacement of the palatal segments, (4) (5) and show a clear tendency to intensify during growth. Graber $^{(6)}$ in his landmark study documented severe three dimensional maxillary collapse in patients with complete cleft lip and palate after surgery, resulting in shortening of the maxillary length and decrease in transverse and vertical dimensions. These patients always present with anterior and posterior cross bites and midface deficiency with a tendency toward Class III malocclusion.

Thus, due to the effects of multiple surgeries in patients with cleft lip and palate, the maxilla is greatly affected in the three planes of space. Therefore, accurate diagnosis is important to localize these defects and accordingly, plan the accurate treatment.

For craniofacial analysis, lateral cephalograms have intrinsic limitations that result in distorted images enlarged in some areas and reduced in others. Two dimensional norms cannot be readily used for three dimensional measurements because of differences in measurements accuracy between the two ${ }^{(7)}$. Cephalometric evaluation of radiographic images taken in the conventional two dimensional system often renders both inaccurate and imprecise measurements ${ }^{(8)}$. Limitations associated with cephalometric radiographs render it difficult to reliably assess deeper craniofacial structures.

A more recent technology catching the attention of the orthodontic community is cone beam computed tomography (CBCT). CBCT is an evolution of the original computed tomography. It allows the orthodontist to assess the patient's hard and soft tissue in three dimensions.

Because of the advances in both CBCT scanners and software designed to manage CBCT data, it is possible to take advantage of CBCT information in a clinical setting. CBCT allows all necessary radiographs to be collected in one scan, which facilitates data acquisition, storage and duplication. (9) $\mathrm{CBCT}$ allows for clinically accurate and reliable three dimensional linear measurements of the craniofacial complex. Moreover, skull orientation during CBCT scanning does not affect the accuracy or the reliability of these measurements.

By constructing a perspective lateral image from CBCT scan, one can duplicate the inherent magnification of a conventional two dimensional lateral cephalogram. (10) Although many studies proved the accuracy of measurements on three dimensional reconstructed images, the accuracy of measurements on synthesized two dimensional images is not yet well approached.

The aim of this study was to compare the angular and linear measurements of the nasomaxillary complex for unilateral cleft lip and palate patients obtained from CBCT synthesized two dimensional lateral cephalograms to those from $\mathrm{CBCT}$ three dimensional reconstructions.

\section{MATERIAL AND METHODS}

This study comprised 10 children (6 boys and 4 girls) with surgically repaired complete unilateral cleft lip and palate (UCLP) ranging in age from 8 to 12 years (mean $=9.8$ years, $\mathrm{SD}=1.5$ years). The subjects were selected from the outpatient clinic of Orthodontic Department and Cleft - Care Center affiliated to the Oral and Maxillofacial Surgery Department, Faculty of Dentistry, Ain Shams University. 
CBCT scans were acquired for all of the patients using i-CAT scanner (Model 17/ 19 series; Imaging Sciences International, Hatfield, PA) the field of view (FOV) used was the portrait mode, which gathers data in extended FOV mode and covers the full skull of $170 \mathrm{~mm}$ in height X $230 \mathrm{~mm}$ in diameter. The scans were made at $(18.54 \mathrm{~mA},(120$ KVP), with a single 360 o rotation and a total scan time of $(8.9 \mathrm{sec})$. the raw images were exported using the i- CAT vision TM V 1.8 software into DICOM 3 multifiles.

The Maximum intensity projections (MIP) were used to optimally visualize anatomic details. In the MIP projections, the three dimensional images are generated by projecting on the visualization plane the voxel with the highest intensity from the view point to plane of projection. In order for the inner structures of the cranium. (E.g. Sella) to be visible in the MIP cephalograms, the parietal bone had to be excised from the CBCT data sets.

The CBCT synthesized image of each subject was saved as JPG image in an output folder on the hard disk after a line representing the $\mathrm{x}$ and $\mathrm{y}$ coordinates was drawn on the corner of each image to measure the magnification that might have occurred in the saved JPG image.

The DICOM images were loaded into materialize mimics software. A customized analysis was created using the dedicated "Three Dimensional Cephalomeric" software module. The customized three dimensional analysis was saved to be used with all the CBCT scans. Segmentation for bone structure followed by $3 \mathrm{D}$ rendering reconstruction was done.

Seven points were created and identified on the three dimensional volumetric model. (Fig 1). The identified landmarks were then refined in the coronal, sagittal and axial tomographic two dimensional slices to insure the right position of the points. Three linear and three angular measurements were determined and calculated by the software.

Using i-CAT Vision TMV 1.8 software, the multiplanar reformatting screen (MPR) - (displaying the volumetric data set in axial, coronal, and sagittal image slices) was used to synthesize the lateral two dimensional cephalograms from the CBCT scans.

Orthodontic digitizing software program Onyx Ceph ${ }^{\circledR}$ version 2.6.24 (142) was used to digitize the seven points on each image. Then the same angular and linear measurements predefined in the data base of the program were measured and calculated automatically by the software program.

\section{Measurements:}

\section{Digitized Points:}

1. ANS

2. PNS

3. Sella $(\mathrm{S})$

4. Nasion $(\mathrm{N})$

5. Point A

6. Incisal point of upper incisor

7. Root apex of upper incisor

\section{Linear measurements ( $\mathrm{mm})$ :}

1. ANS-PNS

2. Maxillary Height.

3. Facial Height.

\section{Angular measurements (degrees):}

1. SNA.

2. UI- SN

3. UI-PP 

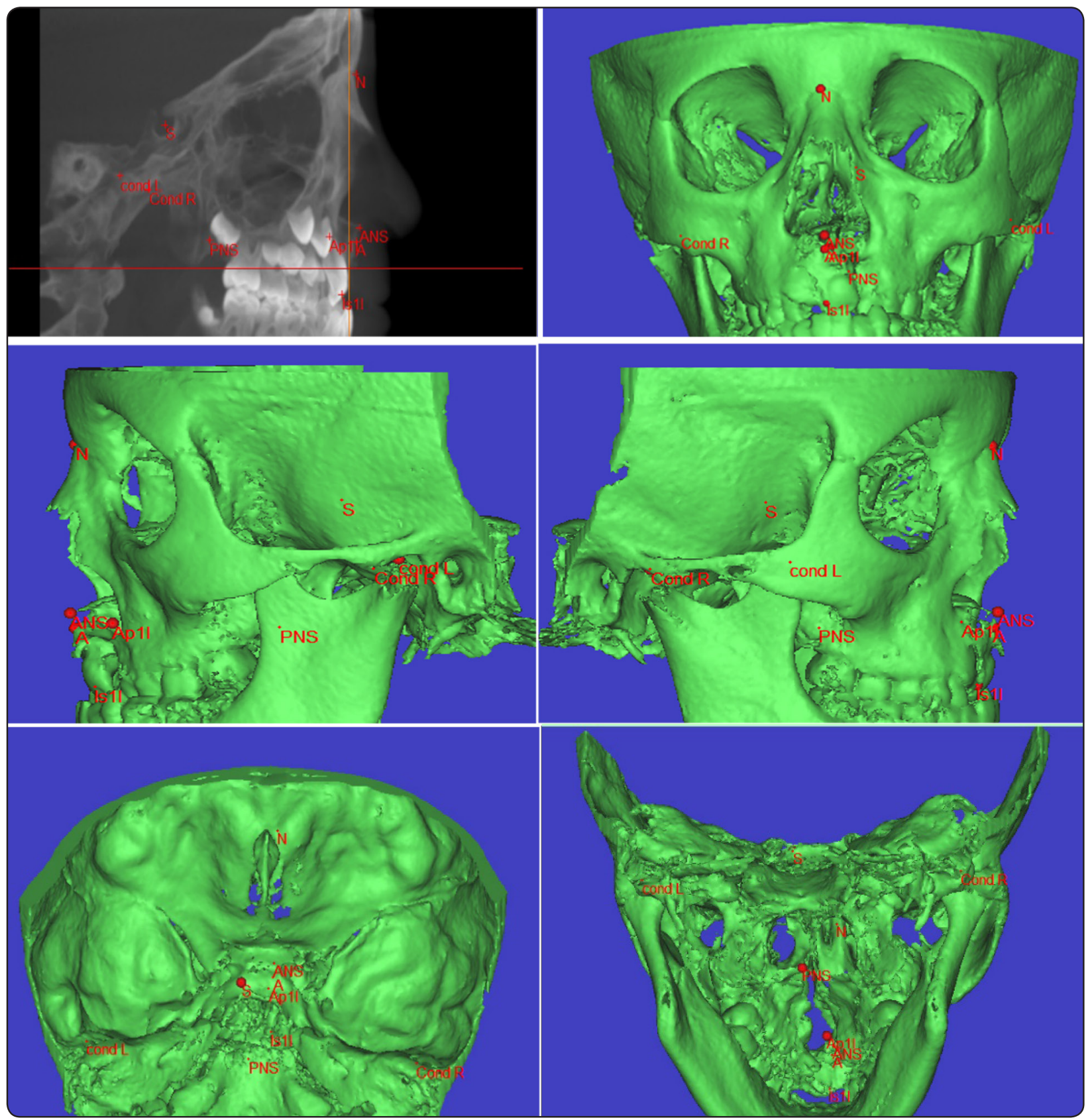

Fig. (1): Seven points were created and identified on the 3D volumetric model.

\section{Statistical analysis}

All measurements were presented into an excel spreadsheet and analyzed using a statistical software package (SPSS version 17.0, Chicago, III) for windows
Descriptive statistics minimum, maximum, mean and standard deviation of the $2 \mathrm{D}$ and $3 \mathrm{D}$ measurements was done.

The difference between the $2 \mathrm{D}$ and the $3 \mathrm{D}$ measurements was statistically assessed using the following statistical tests: 
1) The mean absolute difference MAD and the relative mean absolute difference RMAD was used to assess the difference between the 2D and the 3D measurements.

2) The limit of agreement LOA Bland \&Altman was used at $95 \%$ confidence limit to quantify the size of the difference between the $2 \mathrm{D}$ and the 3D measurements.

3) The concordance correlation coefficient CCC was used at $95 \%$ confidence limit to measure the agreement between the $2 \mathrm{D}$ and $3 \mathrm{D}$ measurements on continuous scales.

\section{RESULTS}

Descriptive statistics minimum, maximum, mean and standard deviation of the $2 \mathrm{D} \& 3 \mathrm{D}$ measurements was shown in table (1).

Table (2) shows a small MAD and RMAD regarding all the measurements except the MaxHeight which was relatively high indicating less agreement than all the other measurements that assure closer agreement between the $2 \mathrm{D}$ and the $3 \mathrm{D}$ measurements.

TABLE (1) Descriptive statistics minimum, maximum, mean and standard deviation of the 2D \& 3D measurement:

\begin{tabular}{|l|c|c|c|c|c|}
\hline \multicolumn{2}{|c|}{} & Minimum & Maximum & Mean & Std. Deviation \\
\hline \multirow{3}{*}{ ANS-PNS } & 3D & 40.30 & 47.60 & 44.00 & 3.32 \\
\cline { 2 - 6 } & 2D & 40.30 & 47.60 & 43.96 & 3.32 \\
\hline \multirow{3}{*}{ Max -Height } & 3D & 4.00 & 9.50 & 6.40 & 2.63 \\
\cline { 2 - 6 } & 2D & 4.00 & 7.90 & 5.74 & 1.77 \\
\hline \multirow{3}{*}{ UI-SN } & 3D & 72.20 & 83.30 & 78.20 & 4.18 \\
\cline { 2 - 7 } & 2D & 72.00 & 83.30 & 78.30 & 4.28 \\
\hline \multirow{3}{*}{ Facial Height } & 3D & 75.90 & 89.80 & 83.12 & 5.74 \\
\cline { 2 - 7 } & 2D & 75.90 & 89.80 & 41.74 & 5.79 \\
\hline \multirow{2}{*}{ UI_PP } & 3D & 33.90 & 45.70 & 41.72 & 4.57 \\
\cline { 2 - 7 } & 2D & 33.90 & 45.70 & 80.84 & 4.57 \\
\hline
\end{tabular}

Std. Deviation: Standard Deviation

TABLE (2) Comparison of the 2D and the 3 D measurements using the mean absolute difference MAD and the relative mean absolute difference RMAD

\begin{tabular}{|c|c|c|c|c|c|c|}
\hline & ANS - PNS & Max -Height & SNA & UI-SN & Facial height & UI_PP \\
\hline MAD & 0.056 & 0.644 & 0.206 & 0.092 & 0.044 & 0.012 \\
\hline RMAD & $0.13 \%$ & $10.61 \%$ & $0.26 \%$ & $0.11 \%$ & $0.11 \%$ & $0.01 \%$ \\
\hline
\end{tabular}


Table (3) illustrates a close agreement between all the 2D and 3D measurements with lesser agreement regarding the Max-Height.
Table (4) confirms excellent agreement or reliability between the $2 \mathrm{D}$ and the $3 \mathrm{D}$ measurements CCC $>0.9$ with only good agreement for the MaxHeight as $\mathrm{CCC}>0.8$

TABLE (3) Comparison of the 2D and the $3 \mathrm{D}$ measurements using the Bland \& Altman Limits of Agreement (LOA) at 95\% confidence limit

\begin{tabular}{|c|c|c|c|c|c|c|}
\hline & ANS - PNS & Max -Height & SNA & UI-SN & Facial height & UI_PP \\
\hline $\begin{array}{c}\text { LOA } \\
\text { Mean Difference }\end{array}$ & -0.056 & -0.644 & 0.082 & -0.092 & -0.044 & -0.004 \\
\hline LL & 0.096 & 1.305 & 0.781 & 0.290 & 0.042 & 0.032 \\
\hline UL & -0.208 & -2.593 & -0.617 & -0.474 & -0.130 & -0.040 \\
\hline
\end{tabular}

LL: Lower Limit

UL: Upper Limit

TABLE (4) Comparison of the 2D and the $3 \mathrm{D}$ measurements using the Concordance Correlation Coefficient at $95 \%$ confidence limit.

\begin{tabular}{|c|c|c|c|c|c|c|}
\hline & ANS - PNS & Max -Height & SNA & UI-SN & facial height & UI_PP \\
\hline CCC & 1.000 & 0.858 & 0.996 & 0.999 & 1.000 & 1.000 \\
\hline LL & 0.997 & 0.584 & 0.978 & 0.996 & 0.999 & 1.000 \\
\hline UL & 1.000 & 0.956 & 0.999 & 1.000 & 1.000 & 1.000 \\
\hline
\end{tabular}

LL: Lower Limit

UL: Upper Limit

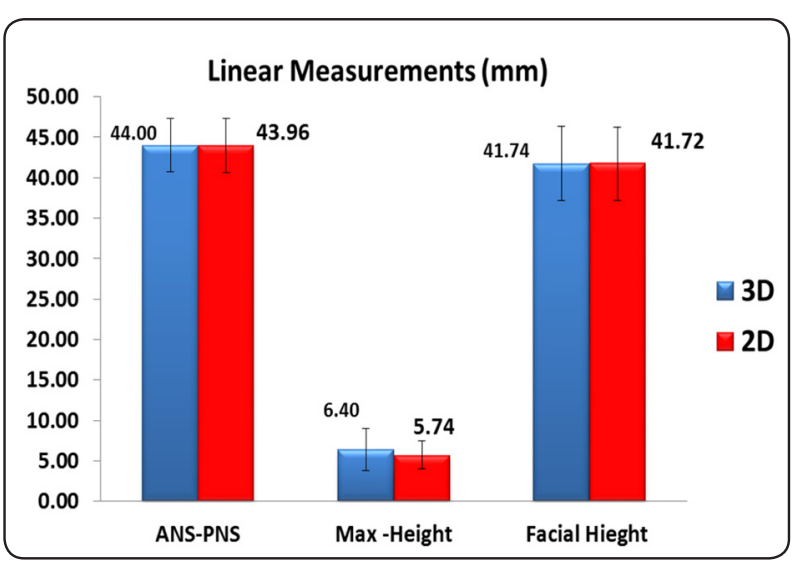

Fig (2) A bar chart of the 2D and 3D linear measurements

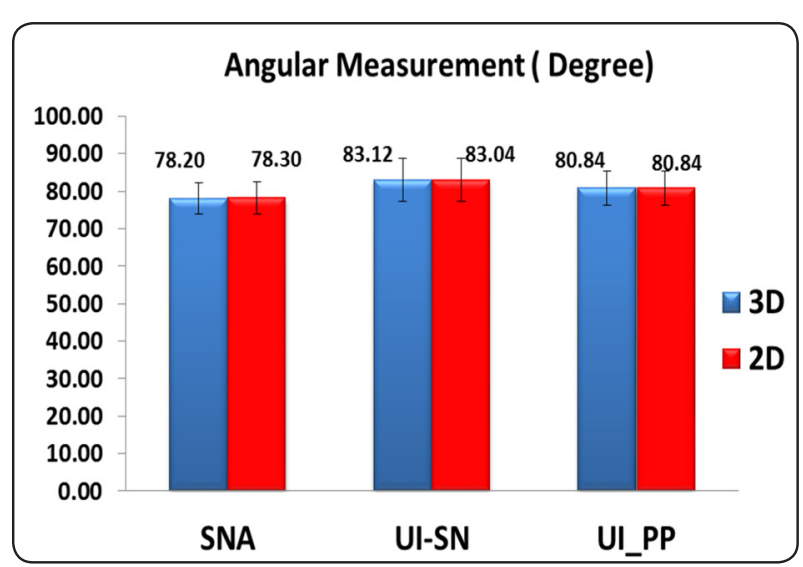

Fig (3) A bar chart of the 2D and 3D Angular measurements 


\section{DISCUSSION}

Lateral cephalograms have intrinsic limitations that results in distorted images, enlarged in some areas and reduced in others. Two dimensional cephalomeric norms cannot be readily used for three- dimensional measurements because of differences in measurement accuracy between the two exams.

When using a gold standard of physical measurements from the dry skulls, cephalometric evaluation of radiographic images taken in the conventional two dimensional system often renders both inaccurate and imprecise measurements. In contrast, the relatively new three dimensional cephalometric system (Sculptor) provides a much more precise evaluation of linear measures and only slightly inaccurate measures that are underestimated by about $1.0 \mathrm{~mm}$. the cephalometric measures from the three dimensional Sculptor provide a much more valuable tool in assessing growth and the effects of orthodontic treatment.

CBCT allows for clinically accurate and reliable three dimensional linear measurements of the craniofacial complex. Moreover, skull orientation during CBCT scanning does not affect the accuracy or the reliability of these measurements.

Although CBCT acquires images in 1:1 perspective, the software used in this study allowed for automatic correction of any magnification that might have accused during saving the scanned image. The magnification was compensated for during computation of all linear measurements. Measurements were reproducible and significantly more accurate than those made with conventional cephalograms in all the three orthogonal planes.

For most two dimensional cephalometric measurements in the sagittal plane, simulated two dimensional lateral cephalometric projections from cone- beam computed tomography (CBCT) proved to be more accurate than conventional digital lateral
cephalograms.Although 2D cephalograms generated from single CBCT basis projections improved the accuracy of cephalometric measurements over conventional cephalometry, there was no additional gain from using ray- sum images generated from the CBCT volumetric data set.

The results of this study show a close agreement between all the two dimensional and three dimensional measurements with lesser agreement regarding the Max-Height.

These findings are in accordance with Hariharan et al ${ }^{(11)}$, Bonfim et $\mathrm{al}^{(12)}$, Cassetta et $\mathrm{al}^{(13)}$ who found that linear and angular measurements from CBCT were similar to measurements from conventional lateral cephalogram. It is important to note that none of these studies used the synthesized two dimensional images from CBCT, nor did any studied cleft patients. The novelty of this work made it not possible to find similar work to compare to.

Contrasting to the results of the present study, Abé-Nickler ${ }^{(14)}$ found no correlation between twodimensional measurements and three-dimensional configuration of the pharyngeal upper airway space in cone-beam computed tomography. This could be due to the volumetric nature of the structure of interest in their study, the pharyngeal airway, which lent a lower validity of two dimensional measurements and resulted in low correlation to three dimensional measurements.

The results of this study show that by constructing a perspective lateral image from an i- CAT CBCT scan, one can replicate the inherent magnification of a conventional two dimensional lateral cephalogram with high accuracy. This image can be used in place of a two dimensional lateral cephalogram for comparison with either normative data or serial records. This measure eliminates the need to take an additional lateral cephalogram once a CBCT scan has been taken, increasing efficiency and reducing ionizing radiation to the patient. 
As treatment progresses, a serial lateral cephalogram can be taken and compared with the perspective image from an earlier CBCT scan at much lower radiation than another CT scan. The eventual standardization of the CBCT as an initial record will allow clinicians to gather significantly more accurate and comprehensive information about the patient while maintaining useful diagnostic tools commonly used by orthodontists.

\section{CONCLUSION}

Two dimensional synthetized image from CBCT is reliable in both linear and angular measurements in cleft patients, with lesser agreement regarding the Max-Height. By constructing a perspective lateral image from $\mathrm{CBCT}$ scan, one can replicate the inherent magnification of a conventional two dimensional lateral cephalogram with high accuracy. This image can be used in place of a two dimensional lateral cephalogram for comparison with either normative data or serial records.

\section{REFERENCES}

1. Jones KL. Smith's recognizable patterns of human malformation, fourth edition, W.B. Saunders Company, Philadelphia, 1988.

2. Bishra SE, De Arrendondo RSM, Vales HP and Jakobsen JR. dentofacial relationships in persons with unoperatic clefts: comparison between three cleft types. Am J Orthod 1985; 87: 481- 507.

3. Filho OG, Ramos AL, and Abdo RC. The influence of unilateral cleft lip and palate on maxillary dental arch morphology. Angle Orthod 1992; 62: 283-290.

4. Mazaheri M, Harding RL, Cooper JA, Meier JA and Jones TS. Changes in arch form and dimensions of cleft patients. Am J Orthod 1971; 60: 19-32.

5. Wada T and Miyazaki T. Growth and changes in maxillary arch form in complete unilateral cleft lip and palate children. Cleft palate J 1975; 12: 115-130.
6. GrabberTM.Acephalometric analysis of the developmental pattern and facial morphology in cleft palate. Angle Orthod 1949; 19: 91-100.

7. Gribel BF, Gribel MN, Fraza o DC, McNamara JA and Manzi FR. Accuracy and reliability of craniometric measurements on lateral cephalometry and 3D measurements on CBCT scans. Angle Orthod 2011; 81: 26-35.

8. Adams GL, Gansky SA, Miller AJ, Harrell WE, and Hatcher DC. Comparison between traditional 2dimensional cephalometry and a 3- dimensional approach on human dry skull. Am. J. Orthod Dentofacial Orthop 2004; 126: 397-409.

9. Sukovic P. Cone beam computed tomography in craniofacial imaging. Orthod Craniofac Res 2003; 6:31-63.

10. Lamichane M, Anderson NK, Rigali PH, Seldin EB and Will LA. Accuracy of reconstructed images from cone- beam computed tomography scans. Am J Orthod Dentofacial Orthop 2009; 136:156. El-156.e6.

11. Hariharan A, Diwakar NR, Jayanthi K, Hema HM, Deepukrishna S, Ghaste SR. The reliability of cephalometric measurements in oral and maxillofacial imaging: Cone beam computed tomography versus twodimensional digital cephalograms. Indian J Dent Res. 2016 Jul-Aug; 27(4):370-377.

12. Bonfm MA, Costa AL, Fuziy A, Ximenez ME, CotrimFerreira FA, Ferreira-Santos RI. Cervical vertebrae maturation index estimates on cone beam CT: 3D reconstructions vs sagittal sections. Dentomaxillofac Radiol. 2016; 45(1):20150162.

13. Cassetta M, Altieri F, Di Giorgio R, Silvestri A. TwoDimensional and Three-Dimensional Cephalometry Using Cone Beam Computed Tomography Scans. J Craniofac Surg. 2015 Jun; 26(4):e311-5.

14. Abé-Nickler MD, Pörtner S, Sieg P, Hakim SG .No correlation between two-dimensional measurements and three-dimensional configuration of the pharyngeal upper airway space in cone-beam computed tomography. Craniomaxillofac Surg. 2017 Mar; 45(3):371-376. 